\title{
Dietary-morphological relationships in fish assemblages of small forested streams in the Bolivian Amazon
}

\author{
Carla Ibañez ${ }^{1-6 a}$, Pablo A. Tedesco ${ }^{2}$, Rémy Bigorne ${ }^{3}$, Bernard Hugueny $^{4}$, Marc Pouilly ${ }^{3}$, Claudia Zepita ${ }^{6}$, \\ José Zubieta $^{5}$ and Thierry Oberdorff ${ }^{1 *}$ \\ ${ }^{1}$ Institut de Recherche pour le Développement (IRD), Département Milieux et Peuplements Aquatiques, Muséum National d’Histoire \\ Naturelle, 43 rue Cuvier, 75231 Paris, France \\ 2 Institut d'Ecologia Aquàtica, Universitat de Girona. Campus de Montilivi, 17071 Girona, Spain \\ 3 Institut de Recherche pour le Développement (IRD), Unidad de Limnologia y Recursos Acuáticos, Universidad Mayor de San Simón, \\ Casilla 5263, Cochabamba, Bolivia \\ ${ }^{4}$ Institut de Recherche pour le Développement (IRD), UMR CNRS 5023, Université de Lyon 1, 43 Bd. du 11 de Novembre 1918, \\ 69622 Villeurbanne Cedex, France \\ 5 Unidad de Limnologia y Recursos Acuáticos, Universidad Mayor de San Simón, Casilla 5263, Cochabamba, Bolivia \\ ${ }^{6}$ Instituto de Ecología - Unidad de Limnología, Universidad Mayor de San Andrés, Casilla 10077, La Paz, Bolivia
}

Received 15 March 2007; Accepted 10 May 2007

\begin{abstract}
We explored the relationships between diet and morphology in 30 fish species from forested tropical streams of the Bolivian Amazon. These species were first assigned to eight broad trophic guilds based on stomach contents analysis. The relationships between diet and morphology were then examined using Redundancy Analysis, after having checked for potential phylogenetical effects. Results show that, independently of any phylogenetic constraints, some of the trophic guilds could be grossly predicted from few relevant morphological attributes (i.e. relative intestinal length, standard length and mouth orientation) and thus suggest a significant link between diet and morphology. In other words, species having similar diet tend to converge to some extent on some morphological attributes. This link was nevertheless rather weak, suggesting that even if morphology may set limits to patterns of resource use, these limits are broad enough to allow fishes changing their choice of prey resources to respond to local biotic and/or abiotic conditions.
\end{abstract}

Key words: Diet / Morphology / Phylogeny / Convergence / Tropical streams / Fishes / Bolivia

Résumé - Relations entre le régime alimentaire et les caractéristiques morphologiques des peuplements de poissons de petits cours d'eau forestiers de l'Amazonie bolivienne. Nous avons analysé les relations entre le régime alimentaire et la morphologie de 30 espèces de poissons présentes dans de petits cours d'eau forestiers de l'Amazonie bolivienne. Après une analyse de leurs contenus stomacaux, les 30 espèces ont été réparties, dans un premier temps, dans huit grandes guildes trophiques. Dans un deuxième temps, après avoir analysé les éventuelles contraintes phylogénétiques, nous avons examiné, par analyse multivariée, les relations entre le régime alimentaire et la morphologie des espèces. Les résultats montrent que, indépendamment de la phylogénie, certaines des guildes trophiques peuvent être prédites d'après quelques attributs morphologiques (i.e. longueur relative de l'intestin, longueur standard et orientation de la bouche). Cela étant, ce lien entre régime alimentaire et caractéristiques morphologiques reste relativement faible, ce qui suggère que même si la morphologie limite les possibilités d'utilisation des ressources, cette limite est assez large pour permettre aux espèces de s'adapter à différentes conditions biotiques ou abiotiques locales.

\section{Introduction}

Seeking to test the ecomorphological hypothesis (i.e. particular interactions between the morphology of organisms and their ecology) in fish assemblages, many studies have significantly related diet to several morphological characteristics of species (Gazt 1979; Kotrschal 1989; Wikramanayake 1990;

\footnotetext{
a Corresponding author:

carla.lunallena@gmail.com; oberdorf@mnhn.fr
}

Winemiller et al. 1995; Piet 1998; Hugueny and Pouilly 1999; Xie et al. 2001; Elliott and Bellwood 2003; Pouilly et al. 2003). For instance, in fishes, gut length clearly distinguish algivores, detritivores and herbivores from carnivores (Paugy 1994; Kramer and Bryant 1995; Delariva and Agostinho 2001; Ward-Campbell et al. 2005). However, relationships between diet and morphology are equivocal since other studies found weak and indistinct results rather relating feeding and morphological variables to local environmental factors and 
resource availability (Grossman 1986; Motta 1988; Douglas and Matthews 1992; Winemiller and Adite 1997; Bellwood et al. 2006). Potential large regional changes can be a source of bias explaining these mixed results since feeding and morphological plasticity can be induced by environmental variability (Wainwright et al. 1991; Wimberger 1992; Hegrenes 2001).

Ecological characteristics of organisms can also be related to their shared evolutionary history. Indeed, species sharing a common ancestor cannot be considered independent in a statistical sense, since it is likely that they are quite similar for the features studied (Felsenstein 1985). Significant relationships between diet and morphology can then be spurious by-products of phylogenetic relatedness between species. However, only a few studies have attempted to control for evolutionary distance between species. For instance, Hugueny and Pouilly (1999) and Pouilly et al. (2003) assessed how their results were affected by taxonomic relatedness (as a surrogate of phylogenetic distances) of the species compared. In both cases, when taxonomic relatedness was factored out, relationships between diet and morphology were still significant.

Here we focus on the relationships between diet and morphology within a local assemblage of 30 species from tropical forested headwater streams of the Bolivian Amazon. Using methods dealing with true phylogenetic information, and avoiding potential regional and environmental effects on species plasticity by working at the local level within sites displaying minimal variations in environmental characteristics, we examine the correlations between diet and relevant morphological variables.

\section{Methods}

\section{Study area}

The study was conducted in five tropical, highly forested, headwater tributaries including 27 sites situated in the upper Rio Chipiriri river catchment of the Bolivian Amazon (total area $<100 \mathrm{~km}^{2}$ ). Annual precipitations within the geographical zone vary between 5000 and $6000 \mathrm{~mm}$, with a rank of mean temperatures between 24 and $26{ }^{\circ} \mathrm{C}$ (Navarro and Maldonado 2002). The five tributaries originated in the same region, were similar in size and environmental characteristics (i.e. physical and water quality characteristics), and were located between the coordinates $16^{\circ} 40^{\prime} \mathrm{S}, 65^{\circ} 25^{\prime} \mathrm{W}$ and $17^{\circ} 00^{\prime} \mathrm{S}, 65^{\circ} 15^{\prime} \mathrm{W}$ at a mean altitude of $270 \mathrm{~m}$. At the basin scale, based on aerial photographs, the percentage of canopy cover along the five tributaries was identical and approximated 100 percent. At the local scale, the 27 sites had overall similar habitat characteristics (mean width $6.95 \mathrm{~m}( \pm 2.26 \mathrm{SD})$, mean depth $0.27 \mathrm{~m}( \pm 0.10 \mathrm{~m}$ $\mathrm{SD})$ and mean velocity $0.18 \mathrm{~m} \mathrm{~s}^{1}( \pm 0.21 \mathrm{SD})$, same regional species pool, and a slight gradient in canopy cover.

\subsection{Fish sampling and habitat description}

Electro-fishing was performed during the dry season from June to October 2004. The 27 sample sites (between 21 and $54.9 \mathrm{~m}$ long reaches) were chosen to encompass complete sets of the characteristic stream form (e.g. pools and riffles). The upstream and downstream edges of the sampled area for each site were blocked by closing nets ( $1 \mathrm{~mm}$ mesh size). Two fishing removals were performed by site, applying a constant fishing effort. Fishes were fixed in formaldehyde $4 \%$ and brought to laboratory for identification to the species level (or the genus level if systematic knowledge was insufficient), counting and weighing.

For each sampled site three environmental descriptors were measured: mean stream width $(\mathrm{m})$, mean stream depth (m) and mean stream flow velocity $(\mathrm{m} / \mathrm{s})$. Width, depth and flow velocity were measured by cross-stream transects at 3-5 $\mathrm{m}$ intervals (depending on the stream size), with sampling points spaced $1 \mathrm{~m}$ apart (for depth and flow velocity). A detailed description of the methodology is given in Tedesco et al. (2007).

\subsection{Fish diet estimation}

The 30 species analyzed in the present study represented $97.4 \%$ of the total number of individuals captured during the study (10 384 over 10660 individuals). These species belonged to 13 families and four orders: one family of Beliniformes, seven families of Characiformes, one family of Perciformes and four families of Siluriformes (Table 1).

Diet estimation was based on stomach contents analysis of adult fishes only. Empty or highly digested stomachs were excluded. The stomach contents were examined with a matting microscope and were separated in five broad categories: substratum (MUD), algae and/or periphyton (ALG), terrestrial vegetation or seeds (VEG), aquatic invertebrate (AIN), terrestrial invertebrates (TIN), and fish (FISH). The substratum category does not correspond to a clearly identified biological feeding resource; but according to Power $(1983,1984)$, fishes can ingest superficial sediment while consuming biological material associated with the substratum. This category was conserved as an indicator of a particular feeding habitat. We used a binocular microscope for most evaluations and a microscope with a Sedgwick-Rafter for algae and detritus materials. Data for these two last categories are qualitative estimates based on material size.

Several methods have been developed for analyzing fish feeding habits (Hyslop 1980; Michel and Oberdorff 1994). Here, we used a slightly modified version of the dominance method (Frost and Went 1940; Tresierra and Culquichicon 1993). This method is based on the size and abundance of prey in the stomach, where each category of food item is allotted a number of points (from 1 to 4 ) proportional to the stomach fullness and the points gained by each food item are summed, indicating the amount or "the real" bulk of each item category. The $\%$ of occurrence of an item in the diet was estimated by divided the number of stomachs that contained that item by the total number of non-empty stomachs analyzed in that particular species.

\subsection{Morphological variables}

The morphological variables were selected based on previous works by Gatz (1979); Watson and Balon (1984); 
Table 1. Taxonomic classification of the 30 studied species.

\begin{tabular}{|c|c|c|c|c|}
\hline Order & Family & Code & & Scientific name \\
\hline Beloniformes & Belonidae & POTEI & Potamorrhaphis eigenmanni & Miranda-Ribeiro, 1915 \\
\hline \multirow[t]{18}{*}{ Characiformes } & Anostomidae & LEOST & Leporinus striatus & Kner, 1858 \\
\hline & Characidae & ASYAB & Astyanax abramis & (Jenyns, 1842) \\
\hline & & ASYLI & Astyanax lineatus & (Perugia, 1891) \\
\hline & & $\mathrm{CHCBO}$ & Characidium bolivianum & Pearson, 1924 \\
\hline & & GEPCH & Gephyrocharax chaparae & Fowler, 1940 \\
\hline & & HEMLU & Hemigrammus cf. lunatus & Durbin, 1918 \\
\hline & & HEMSP & Hemibrycon sp. & \\
\hline & & KNOSP & Knodus sp. & \\
\hline & & MOEOL & Moenkhausia oligolepis & (Günther, 1864) \\
\hline & & PHEPE & Phenacogaster pectinatus & (Cope, 1870) \\
\hline & & SERSP & Serrapinnus sp. & \\
\hline & & TYTMA & Tyttocharax cf. madeirae & Fowler, 1913 \\
\hline & Curimatidae & STIDO & Steindachnerina dobula & (Günther, 1868) \\
\hline & & STIGU & Steindachnerina guentheri & (Eigenmann \& Eigenmann, 1889) \\
\hline & Erythrinidae & HOPMA & Hoplias malabaricus & (Bloch, 1794) \\
\hline & Lebiasinidae & PYRVI & Pyrrhulina vittata & Regan, 1912 \\
\hline & Parodontidae & PARBU & Parodon cf. buckleyi & Boulenger, 1887 \\
\hline & Prochilodontidae & PRONI & Prochilodus nigricans & Spix \& Agassiz, 1829 \\
\hline \multirow[t]{4}{*}{ Perciformes } & Cichlidae & APISP & Apistogramma sp. & \\
\hline & & CIABO & Cichlasoma boliviense & Kullander, 1983 \\
\hline & & CRISE & Crenicichla cf. semicincta & Steindachner, 1892 \\
\hline & & SATJU & Satanoperca jurupari & (Heckel, 1840) \\
\hline \multirow[t]{7}{*}{ Siluriformes } & Callichthyidae & CORSP & Corydoras spp. & \\
\hline & Heptapteridae & IMPST & Imparfinis cf. stictonotus & (Fowler, 1940) \\
\hline & & RHAQE & Rhamdia quelen & (Quoy \& Gaimard, 1824) \\
\hline & Loricariidae & ANCSP & Ancistrus spp. & \\
\hline & & HYPCO & Hypostomus gr. cochliodon & Kner, 1854 \\
\hline & & RINLA & Rineloricaria lanceolata & (Günther, 1868) \\
\hline & Trichomycteridae & ITUAM & Ituglanis cf. amazonicus & (Steindachner, 1882) \\
\hline
\end{tabular}

Winemmiller (1991); Kramer and Bryant (1995); Hugueny and Pouilly (1999) and Pouilly et al. (2003). Six ecomorphological variables were measured on 5 to 70 individuals for each species, depending on the number of individuals captured (Table 2). Average values of these variables were computed (Table 3).

In order to minimize the influence of body size on the four continuous morphological variables (Table 2), we regressed log-transformed variables against log-Standard Length and used the residuals values for subsequent Redundancy analysis (RDA) (Schneider et al. 1999).

\subsection{Phylogenetic effect}

The degrees of freedom in statistical tests may be inflated due to phylogenetic bias (non-independence of observations). In order to assess the phylogenetic effect in diet-morphology relationships, we compiled a synthetic evolutionary tree using data from Diogo (2003), Malabarba et al. (1998) and the "Tree of Life Web Project" (1995) (http: //tolweb.org) that represents phylogenetic relationships among the genera studied (Fig. 1). We then calculated patristic distances between genera from cytochrome b sequences downloaded from GenBank (860 bp; http://www.ncbi.nlm.nih.gov/Genbank/) and using the synthetic evolutionary tree as a constraint under
PAUP 4.0b10 (Swofford 2002) through the neighbour-joining method.

Based on the patristic distances, we evaluated the phylogenetic effect on our data set by applying a Moran's I statistic (Moran 1948). This autocorrelation index was calculated for 10 classes of patristic phylogenetic distances (Appendix 1). Positive values of Moran's I coefficients show that phylogenetically closer species are more similar than the others for the considered character.

The index of Moran is based on average values and is thus not very sensitive to aberrant values and takes the following form:

$$
\frac{n}{m} \frac{\sum_{i} \sum_{j} W_{i j}\left(Z_{i}-\bar{z}\right)\left(Z_{j}-\bar{z}\right)}{\sum_{i}(Z-\bar{z})^{2}}
$$

where:

$Z_{I}=$ average value of the considered variable

$i=$ unit of reference

$j=$ units close to the item " $i$ ", defined by the matrix $W i j$

$n=$ total number of individuals in the sample $\left(\sum i\right)$

$m=$ total number of pairs of neighbors $\left(\sum i \sum j W i j\right)$

$W i j=$ weighing matrix.

Under the hypothesis of phylogenetic inertia we expect a positive autocorrelation between related species, this autocorrelation decreasing toward null values as phylogenetic distance between species increases. When this situation occurs several 
Table 2. Description of species traits and the functions they describe.

\begin{tabular}{|c|c|c|c|}
\hline Functional trait & Code & Function & Reference \\
\hline $\begin{array}{l}\text { Standard length: Distance from the tip of the } \\
\text { snout to the last vertebra }\end{array}$ & $\mathbf{S L}$ & $\begin{array}{l}\text { No specific function, but could inform on } \\
\text { prey size }\end{array}$ & $\begin{array}{l}\text { Hugueny \& Pouilly (1999), Chan } \\
\text { (2001), Pouilly et al. (2003). }\end{array}$ \\
\hline Eye diameter & ED & $\begin{array}{l}\text { Inform about the fish visual acuity. } \\
\text { Important factor for the search for food. }\end{array}$ & Gatz (1979), Piet (1998) \\
\hline $\begin{array}{l}\text { Head length: Distance from the tip of the snout } \\
\text { to the posterior extension of the operculum }\end{array}$ & HEAL & $\begin{array}{l}\text { Fishes with relatively larger head were found } \\
\text { to consume larger prey }\end{array}$ & Gatz (1979), Watson \& Balon (1984) \\
\hline $\begin{array}{l}\text { Mouth width: Horizontal distance measured } \\
\text { among limit them ends of the mouth }\end{array}$ & MOWI & Maximal prey size & Gatz (1979) \\
\hline $\begin{array}{l}\text { Mouth orientation (code): } 1 \text { - dorsal; } 2 \text { - } \\
\text { terminal; } 3 \text { - oblique; } 4 \text { - ventral }\end{array}$ & MORI & Method of food acquisition & Gatz (1979) \\
\hline $\begin{array}{l}\text { Gut length: Distance from the beginning of the } \\
\text { esophagus to anus }\end{array}$ & GUTL & Informs on the fish trophic status & Gatz (1979) \\
\hline
\end{tabular}

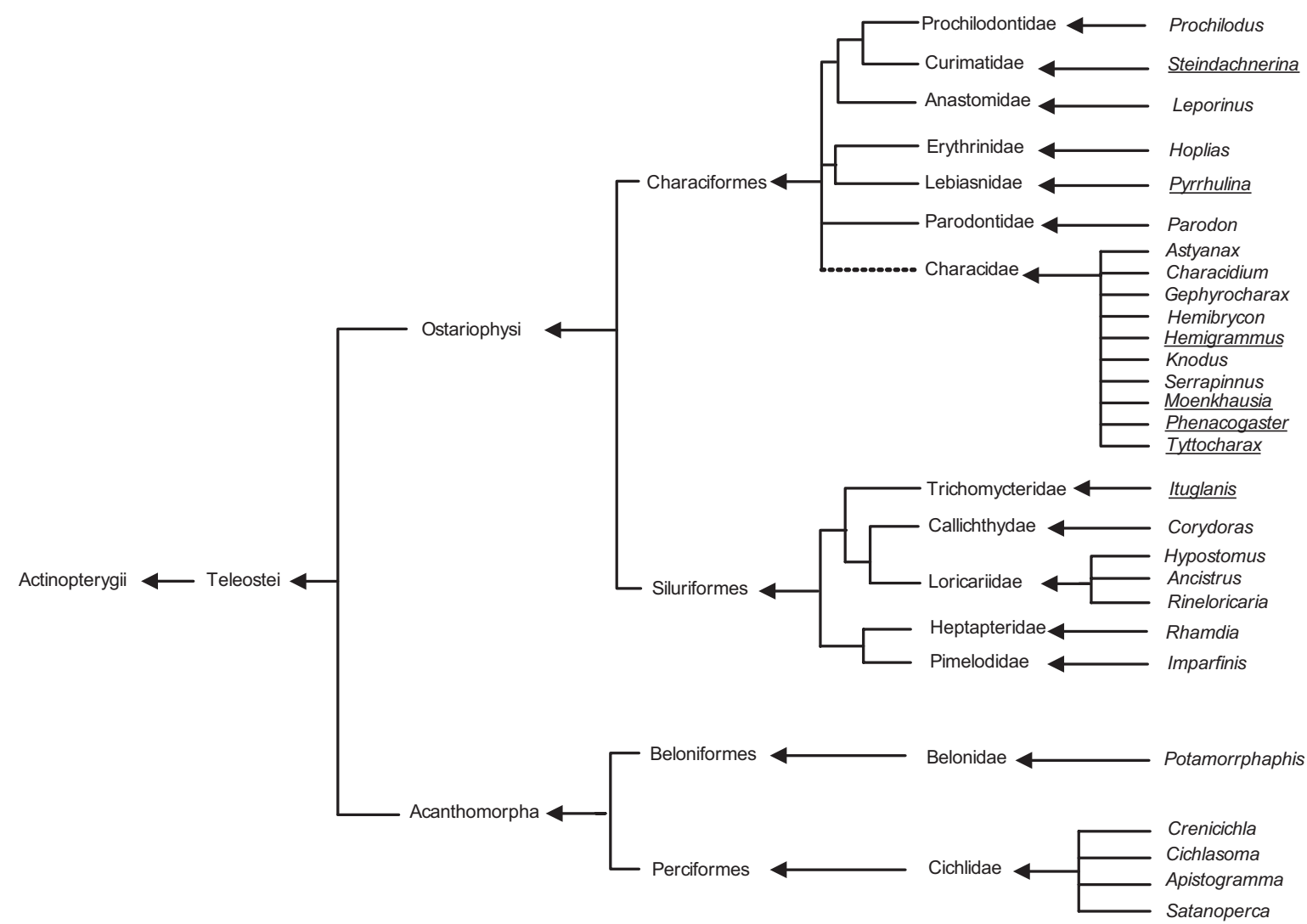

Fig. 1. Hypothetic phylogenetic tree for the 28 genera encountered in our study. The tree is based on compiled data from Diogo (2003), Malabarba et al. (1998) and The Tree of Life Web Project (1995) (http://tolweb.org). Patristic distances between 21 over the 28 genera were then calculated using cytochrome b sequences available in GenBank. The seven genera not included in the calculation of patristic distances are underlined.

methods are available (Harvey and Pagel 1991) to "remove" the phylogenetic autocorrelation. However, phylogenetic inertia was observed for none of our variables and hence no method accounting for phylogenetic relatedness was used.

\subsection{Statistical analyses}

Diet breadth was calculated by using Levin's standardized index: $B_{i}=\left[\left(\sum_{j} P_{i j}^{2}\right)^{-1}-1\right](n-1)^{-1}$ where $P_{i j}$ is the proportion of prey $j$ in the diet of predator $i$ and $n$ is the number of prey categories (Hurlbert 1978). This index ranges from 0 to 1 , low values indicating diets dominated by a few prey items (i.e. specialist species), and high values indicating generalist diets (Gibson and Ezzi 1987; Xie et al. 2001; Pouilly et al. 2003).

In order to identify trophic guilds, we used the species food item matrix (elaborated using our modified version of the dominance method; Table 4) to compute a $\chi^{2}$ distance between species. Distance values were then clustered using unweighted pair group method of arithmetic averages (UPGMA) algorithm (Legendre and Legendre 1998). 
Table 3. Mean values of the six morphological attributes: Standard length (SL; in mm); eye diameter (ED; mm); head length (HEAL; in mm); mouth width (MOWI; mm); mouth orientation (MORI; categorical variable, see Table 2) and gut length (GUTL, mm). Values in parentheses correspond to range values for standard length and to standard deviation values for the other morphological parameters except MORI.

\begin{tabular}{|c|c|c|c|c|c|c|c|}
\hline Species & $\mathbf{N}$ & SL & ED & HEAL & MOWI & MORI & GUTL \\
\hline Ancistrus spp. & 84 & $\begin{array}{c}90 \\
(61-150)\end{array}$ & $\begin{array}{c}5 \\
(0.8)\end{array}$ & $\begin{array}{c}26 \\
(6.5)\end{array}$ & $\begin{array}{c}14 \\
(4.1)\end{array}$ & 4 & $\begin{array}{c}287 \\
(396)\end{array}$ \\
\hline Apistogramma sp. & 432 & $\begin{array}{c}31 \\
(18-46)\end{array}$ & $\begin{array}{c}3 \\
(0.4)\end{array}$ & $\begin{array}{c}11 \\
(1.8)\end{array}$ & $\begin{array}{c}3 \\
(0.7)\end{array}$ & 2 & $\begin{array}{c}34 \\
(7.3)\end{array}$ \\
\hline Astyanax abramis & 499 & $\begin{array}{c}74 \\
(45-109)\end{array}$ & $\begin{array}{c}6 \\
(0.9)\end{array}$ & $\begin{array}{c}21 \\
(3.2)\end{array}$ & $\begin{array}{c}6 \\
(1.2)\end{array}$ & 1 & $\begin{array}{c}60 \\
(16.2)\end{array}$ \\
\hline Astyanax lineatus & 72 & $\begin{array}{c}73 \\
(48-121)\end{array}$ & $\begin{array}{c}6 \\
(0.9)\end{array}$ & $\begin{array}{c}21 \\
(4.6)\end{array}$ & $\begin{array}{c}6 \\
(1.7)\end{array}$ & 1 & $\begin{array}{c}82 \\
(9.3)\end{array}$ \\
\hline Characidium bolivianum & 126 & $\begin{array}{c}52 \\
(29-69)\end{array}$ & $\begin{array}{c}3 \\
(1.6)\end{array}$ & $\begin{array}{c}13 \\
(1.9)\end{array}$ & $\begin{array}{c}2 \\
(0.4)\end{array}$ & 2 & $\begin{array}{c}26 \\
(3.4)\end{array}$ \\
\hline Cichlasoma boliviense & 79 & $\begin{array}{c}69 \\
(51-138)\end{array}$ & $\begin{array}{c}7 \\
(0.8)\end{array}$ & $\begin{array}{c}25 \\
(5.6)\end{array}$ & $\begin{array}{c}8 \\
(2.8)\end{array}$ & 2 & $\begin{array}{c}102 \\
(42.7)\end{array}$ \\
\hline Corydoras spp. & 109 & $\begin{array}{c}51 \\
(41-65)\end{array}$ & $\begin{array}{c}3 \\
(0.3)\end{array}$ & $\begin{array}{c}15 \\
(1.4)\end{array}$ & $\begin{array}{c}3 \\
(0.6)\end{array}$ & 3 & $\begin{array}{l}28 \\
(6)\end{array}$ \\
\hline Crenicichla $\mathrm{cf}$. semicincta & 209 & $\begin{array}{c}89 \\
(60-199)\end{array}$ & $\begin{array}{c}6 \\
(1.2)\end{array}$ & $\begin{array}{c}33 \\
(10.9)\end{array}$ & $\begin{array}{c}10 \\
(4.5)\end{array}$ & 2 & $\begin{array}{c}86 \\
(43.3)\end{array}$ \\
\hline Gephyrocharax chaparae & 79 & $\begin{array}{c}46 \\
(33-55)\end{array}$ & $\begin{array}{c}3 \\
(0.4)\end{array}$ & $\begin{array}{l}11 \\
(1)\end{array}$ & $\begin{array}{c}3 \\
(0.4)\end{array}$ & 1 & $\begin{array}{c}27 \\
(4.8)\end{array}$ \\
\hline Hemibrycon sp. & 80 & $\begin{array}{c}62 \\
(42-85)\end{array}$ & $\begin{array}{c}5 \\
(0.8)\end{array}$ & $\begin{array}{c}16 \\
(3.2)\end{array}$ & $\begin{array}{c}4 \\
(1.2)\end{array}$ & 1 & $\begin{array}{c}58 \\
(11.4)\end{array}$ \\
\hline Hemigrammus cf. lunatus & 191 & $\begin{array}{c}26 \\
(21-33)\end{array}$ & $\begin{array}{c}3 \\
(0.3\end{array}$ & $\begin{array}{c}8 \\
(0.6)\end{array}$ & $\begin{array}{c}2 \\
(0.3)\end{array}$ & 1 & $\begin{array}{c}19 \\
(4.6)\end{array}$ \\
\hline Hoplias malabaricus & 33 & $\begin{array}{c}137 \\
(69-212)\end{array}$ & $\begin{array}{c}5 \\
(1)\end{array}$ & $\begin{array}{c}48 \\
(9.5)\end{array}$ & $\begin{array}{c}16 \\
(3.9)\end{array}$ & 1 & $\begin{array}{c}101 \\
(31.4)\end{array}$ \\
\hline Hypostomus gr. cochliodon & 23 & $\begin{array}{c}55 \\
(34-75)\end{array}$ & $\begin{array}{c}4 \\
(0.6)\end{array}$ & $\begin{array}{c}16 \\
(3.4)\end{array}$ & $\begin{array}{l}7 \\
(3)\end{array}$ & 4 & $\begin{array}{c}560 \\
(156)\end{array}$ \\
\hline Imparfinis cf. stictonotus & 17 & $\begin{array}{c}36 \\
(26-45)\end{array}$ & $\begin{array}{c}1 \\
(0.2)\end{array}$ & $\begin{array}{c}9 \\
(1.2)\end{array}$ & $\begin{array}{c}4 \\
(0.5)\end{array}$ & 2 & $\begin{array}{c}16 \\
(2.4)\end{array}$ \\
\hline Ituglanis cf. amazonicus & 41 & $\begin{array}{c}49 \\
(41-88)\end{array}$ & $\begin{array}{c}2 \\
(0.4)\end{array}$ & $\begin{array}{c}8 \\
(1.2)\end{array}$ & $\begin{array}{c}4 \\
(0.6)\end{array}$ & 2 & $\begin{array}{c}20 \\
(5.6)\end{array}$ \\
\hline Knodus sp. & 23 & $\begin{array}{c}30 \\
(24-37)\end{array}$ & $\begin{array}{c}3 \\
(0.3)\end{array}$ & $\begin{array}{c}8 \\
(0.7)\end{array}$ & $\begin{array}{c}2 \\
(0.5)\end{array}$ & 1 & $\begin{array}{c}31 \\
(7.2)\end{array}$ \\
\hline Leporinus striatus & 96 & $\begin{array}{c}106 \\
(75-148)\end{array}$ & $\begin{array}{c}6 \\
(0.7)\end{array}$ & $\begin{array}{c}28 \\
(3.5)\end{array}$ & $\begin{array}{c}6 \\
(1.6)\end{array}$ & 3 & $\begin{array}{c}132 \\
(36.5)\end{array}$ \\
\hline Moenkhausia oligolepis & 274 & $\begin{array}{c}52 \\
(36-68)\end{array}$ & $\begin{array}{c}5 \\
(0.5)\end{array}$ & $\begin{array}{c}15 \\
(1.8)\end{array}$ & $\begin{array}{c}4 \\
(0.6)\end{array}$ & 1 & $\begin{array}{c}58 \\
(11.3)\end{array}$ \\
\hline Parodon cf. buckleyi & 41 & $\begin{array}{c}97 \\
(63-131)\end{array}$ & $\begin{array}{c}4 \\
(0.8)\end{array}$ & $\begin{array}{c}21 \\
(3.6)\end{array}$ & $\begin{array}{c}6 \\
(1.2)\end{array}$ & 4 & $\begin{array}{c}115 \\
(15.9)\end{array}$ \\
\hline Phenacogaster pectinatus & 269 & $\begin{array}{c}36 \\
(26-49)\end{array}$ & $\begin{array}{c}3 \\
(0.4)\end{array}$ & $\begin{array}{c}9 \\
(1.1)\end{array}$ & $\begin{array}{c}2 \\
(0.3)\end{array}$ & 1 & $\begin{array}{c}17 \\
(3.1)\end{array}$ \\
\hline Potamorrhaphis eigenmanni & 10 & $\begin{array}{c}164 \\
(142-201)\end{array}$ & $\begin{array}{c}6 \\
(0.1)\end{array}$ & $\begin{array}{c}61 \\
(7.4)\end{array}$ & $\begin{array}{c}4 \\
(0.9)\end{array}$ & 2 & $\begin{array}{c}66 \\
(8.7)\end{array}$ \\
\hline Prochilodus nigricans & 34 & $\begin{array}{c}169 \\
(137-202)\end{array}$ & $\begin{array}{c}10 \\
(0.8)\end{array}$ & $\begin{array}{c}53 \\
(5.4)\end{array}$ & $\begin{array}{c}22 \\
(2.5)\end{array}$ & 2 & $\begin{array}{c}579 \\
(235)\end{array}$ \\
\hline Pyrrhulina vittata & 93 & $\begin{array}{c}30 \\
(22-36)\end{array}$ & $\begin{array}{c}3 \\
(0.3)\end{array}$ & $\begin{array}{c}8 \\
(1.1)\end{array}$ & $\begin{array}{c}2 \\
(0.3)\end{array}$ & 1 & $\begin{array}{c}22 \\
(3.8)\end{array}$ \\
\hline Rhamdia quelen & 140 & $\begin{array}{c}120 \\
(67-213)\end{array}$ & $\begin{array}{c}4 \\
(1)\end{array}$ & $\begin{array}{c}31 \\
(8.7)\end{array}$ & $\begin{array}{c}16 \\
(5.4)\end{array}$ & 2 & $\begin{array}{c}158 \\
(61.4)\end{array}$ \\
\hline Rineloricaria lanceolata & 24 & $\begin{array}{c}88 \\
(71-106)\end{array}$ & $\begin{array}{c}3 \\
(0.3)\end{array}$ & $\begin{array}{c}14 \\
(1.8)\end{array}$ & $\begin{array}{c}6 \\
(2.3)\end{array}$ & 4 & $\begin{array}{c}272 \\
(58.2)\end{array}$ \\
\hline Satanoperca jurupari & 12 & $\begin{array}{c}85 \\
(71-130)\end{array}$ & $\begin{array}{c}8 \\
(0.5)\end{array}$ & $\begin{array}{c}32 \\
(6.1)\end{array}$ & $\begin{array}{c}11 \\
(2.5)\end{array}$ & 2 & $\begin{array}{c}93 \\
(20.2)\end{array}$ \\
\hline Serrapinnus sp. & 59 & $\begin{array}{c}28 \\
(24-35)\end{array}$ & $\begin{array}{c}3 \\
(0.2)\end{array}$ & $\begin{array}{c}7 \\
(0.7)\end{array}$ & $\begin{array}{c}2 \\
(0.2)\end{array}$ & 2 & $\begin{array}{c}25 \\
(3.4)\end{array}$ \\
\hline Steindachnerina dobula & 57 & $\begin{array}{c}98 \\
(58-138)\end{array}$ & $\begin{array}{c}6 \\
(1.3)\end{array}$ & $\begin{array}{c}28 \\
(6.9)\end{array}$ & $\begin{array}{c}9 \\
(1.8)\end{array}$ & 3 & $\begin{array}{l}1590 \\
(497)\end{array}$ \\
\hline Steindachnerina guentheri & 20 & $\begin{array}{c}75 \\
(55-95)\end{array}$ & $\begin{array}{c}6 \\
(0.8)\end{array}$ & $\begin{array}{c}21 \\
(3.1)\end{array}$ & $\begin{array}{c}7 \\
(1.5)\end{array}$ & 3 & $\begin{array}{l}1188 \\
(285)\end{array}$ \\
\hline Tyttocharax cf. madeirae & 159 & $\begin{array}{c}15 \\
(11-18) \\
\end{array}$ & $\begin{array}{c}2 \\
(0.3) \\
\end{array}$ & $\begin{array}{c}4 \\
(0.6) \\
\end{array}$ & $\begin{array}{c}1 \\
(0.2) \\
\end{array}$ & 1 & $\begin{array}{c}7 \\
(1.2) \\
\end{array}$ \\
\hline
\end{tabular}


Table 4. Diet composition and diet breadth (Lewin's index, B) of the 30 species studied. The diet composition is expressed as 1 ) values obtained using the dominance method (DM) and 2) the \% of occurrence of each food category in the non-empty stomachs (\% OC): Terrestrial invertebrates (TIN); aquatic invertebrate (AIN); algae (ALG); substratum (MUD); terrestrial vegetation or seeds (VEG) and fish (FISH).

\begin{tabular}{|c|c|c|c|c|c|c|c|c|c|c|c|c|c|c|}
\hline \multirow{2}{*}{ Species } & \multirow{2}{*}{$\begin{array}{c}\text { Number of } \\
\text { stomachs }\end{array}$} & \multicolumn{2}{|c|}{ TIN } & \multicolumn{2}{|c|}{ AIN } & \multicolumn{2}{|c|}{ ALG } & \multicolumn{2}{|c|}{ MUD } & \multicolumn{2}{|c|}{ VEG } & \multicolumn{2}{|c|}{ FISH } & \multirow{2}{*}{$B$} \\
\hline & & \multicolumn{12}{|c|}{$(\mathrm{DM})(\%$ OC) (DM) (\% OC) (DM) ((\% OC) (DM) (\% OC) (DM) (\% OC) (DM) (\% OC) } & \\
\hline Piscivorous & & & & & & & & & & & & & & \\
\hline $\begin{array}{l}\text { Hoplias malabaricus } \\
\text { Herbivorous }\end{array}$ & 17 & 0 & 0 & 1.12 & 0.47 & 0 & 0 & 0.06 & 0.06 & 0.88 & 0.65 & 1.65 & 0.47 & 0.32 \\
\hline Parodon cf. buckleyi & 17 & 0 & 0 & 0.06 & 0.06 & 0 & 0 & 0.24 & 0.24 & 1.94 & 1 & 0 & 0 & 0.01 \\
\hline Leporinus striatus & 27 & 0.27 & 0.23 & 0.41 & 0.32 & 0.45 & 0.18 & 0.23 & 0.14 & 3.27 & 1 & 0 & 0 & 0.03 \\
\hline Prochilodus nigricans & 16 & 0 & 0 & 0 & 0 & 0.06 & 0.06 & 0.5 & 0.44 & 1.19 & 0.94 & 0 & 0 & 0.06 \\
\hline Astyanax lineatus & 5 & 0.8 & 0.4 & 0.4 & 0.2 & 1.4 & 0.4 & 0 & 0 & 3.6 & 1 & 0 & 0 & 0.09 \\
\hline Rhamdia quelen & 26 & 0.73 & 0.28 & 0.62 & 0.36 & 0.38 & 0.12 & 0.54 & 0.16 & 2.31 & 0.88 & 0.38 & 0.12 & 0.12 \\
\hline Astyanax abramis & 67 & 1.24 & 0.57 & 0.51 & 0.32 & 0.24 & 0.22 & 0.18 & 0.16 & 2.94 & 0.91 & 0 & 0 & 0.12 \\
\hline Omnivorous & & & & & & & & & & & & & & \\
\hline Crenicichla cf. semicincta & 16 & 0.63 & 0.31 & 1.25 & 0.38 & 0 & 0 & 0.06 & 0.06 & 0.81 & 0.5 & 0.56 & 0.19 & 0.37 \\
\hline $\begin{array}{l}\text { Cichlasoma boliviense } \\
\text { Terrestrial invertivorous }\end{array}$ & 14 & 1.07 & 0.29 & 0.29 & 0.14 & 0.64 & 0.21 & 0.07 & 0.07 & 0.43 & 0.36 & 0.64 & 0.21 & 0.57 \\
\hline $\begin{array}{l}\text { Potamorrhaphis eigenmanni } \\
\text { General invertivorous }\end{array}$ & 10 & 2.7 & 1 & 0.1 & 0.1 & 0 & 0 & 0 & 0 & 0 & 0 & 0.7 & 0.2 & 0.02 \\
\hline Hemibrycon sp. & 25 & 3 & 1 & 0.5 & 0.27 & 0 & 0 & 0 & 0 & 0.46 & 0.15 & 0 & 0 & 0.02 \\
\hline Pyrrhulina vittata & 29 & 1.31 & 0.76 & 1.79 & 0.86 & 0.14 & 0.03 & 0 & 0 & 0 & 0 & 0 & 0 & 0.15 \\
\hline Hemigrammus cf. lunatus & 25 & 1.35 & 0.69 & 1.92 & 0.85 & 0 & 0 & 0 & 0 & 0 & 0 & 0 & 0 & 0.14 \\
\hline Gephyrocharax chaparae & 28 & 1.71 & 0.96 & 1.86 & 0.86 & 0 & 0 & 0 & 0 & 0.14 & 0.04 & 0 & 0 & 0.17 \\
\hline $\begin{array}{l}\text { Moenkhausia oligolepis } \\
\text { Aquatic invertivorous }\end{array}$ & 30 & 2.33 & 0.97 & 1.03 & 0.67 & 0.7 & 0.2 & 0.07 & 0.03 & 1.7 & 0.63 & 0 & 0 & 0.26 \\
\hline Corydoras spp. & 22 & 0 & 0 & 3.04 & 1 & 0.43 & 0.3 & 0 & 0 & 0.43 & 0.17 & 0 & 0 & 0.03 \\
\hline Phenacogaster pectinatus & 64 & 0.78 & 0.44 & 2.73 & 0.98 & 0.09 & 0.09 & 0.03 & 0.03 & 0 & 0.08 & 0 & 0 & 0.04 \\
\hline Imparfinis cf. stictonotus & 12 & 0.67 & 0.33 & 2.58 & 1 & 0 & 0 & 0 & 0 & 0 & 0 & 0 & 0 & 0.03 \\
\hline Serrapinnus sp. & 21 & 0.27 & 0.23 & 1.77 & 1 & 0.18 & 0.14 & 0 & 0 & 0 & 0 & 0 & 0 & 0.02 \\
\hline Knodus sp. & 20 & 0.05 & 0.05 & 2.63 & 0.79 & 0.32 & 0.16 & 0 & 0 & 0 & 0.21 & 0 & 0 & 0.01 \\
\hline Tyttocharax cf. madeirae & 29 & 0.24 & 0.21 & 3.52 & 1 & 0 & 0 & 0 & 0 & 0 & 0 & 0 & 0 & 0 \\
\hline Ituglanis cf. amazonicus & 10 & 0 & 0 & 2.3 & 1 & 0 & 0 & 0 & 0 & 0 & 0 & 0 & 0 & 0 \\
\hline Satanoperca jurupari & 7 & 0 & 0 & 1.86 & 1 & 0 & 0 & 0 & 0 & 0 & 0 & 0 & 0 & 0 \\
\hline Characidium bolivianum & 29 & 0.03 & 0.03 & 3.53 & 1 & 0 & 0 & 0 & 0 & 0 & 0 & 0 & 0 & 0 \\
\hline $\begin{array}{l}\text { Apistogramma sp. } \\
\text { Mud feeders }\end{array}$ & 30 & 0 & 0.03 & 2.47 & 0.97 & 0.07 & 0.07 & 0 & 0 & 0 & 0 & 0.07 & 0.03 & 0 \\
\hline Steindachnerina dobula & 25 & 0 & 0 & 0 & 0 & 0.88 & 0.24 & 3.44 & 1 & 0 & 0 & 0 & 0 & 0.02 \\
\hline Steindachnerina guentheri & 8 & 0 & 0 & 0 & 0 & 0 & 0 & 4 & 1 & 0 & 0 & 0 & 0 & 0 \\
\hline $\begin{array}{l}\text { Rineloricaria lanceolata } \\
\text { Algivorous }\end{array}$ & 10 & 0 & 0 & 0 & 0 & 0 & 0 & 3.67 & 0.9 & 0 & 0 & 0 & 0 & 0 \\
\hline Hypostomus gr. cochliodon & 15 & 0 & 0 & 0 & 0 & 4 & 1 & 3 & 1 & 0 & 0 & 0 & 0 & 0.16 \\
\hline $\begin{array}{l}\text { Ancistrus spp. } \\
\text { Species VEG FISH }\end{array}$ & 17 & 0 & 0 & 0 & 0 & 4 & 1 & 3 & 1 & 0 & 0 & 0 & 0 & 0.16 \\
\hline
\end{tabular}

Fish diet/morphology relationships were investigated using Redundancy Analysis (RDA) (CANOCO version 4.0; Ter Braak and Smilaurer 1998). RDA is a constrained ordination process (a canonical version of principal component analysis, PCA). The ordination seeks the axes that are best explained by a linear combination of explanatory variables. Each axis is thus a linear combination (i.e. a multiple regression model) of all explanatory variables. Examination of the canonical coefficients (i.e. the regression coefficients of the models) for the explanatory variables on each axis highlights the most important variables in explaining the different axes. When applied to species diet data, the component axes resulting from RDA are interpretable in terms of differences in species' diet. Thus, the component axes in RDA plots represent the distribution of species based on their diet and constrained by the explanatory variables (i.e. the morphological variables expected to be directly linked to diet). The statistical significance of the diet-morphology correlations extracted from the RDA was estimated by a Monte Carlo permutation test (1000 simulations) (Makarenkov and Legendre 2002; Legendre et al. 2005).

\section{Results}

\subsection{Diets analysis and trophic guilds}

Empty stomachs were found in $11.1 \%$ of a total of 840 analyzed individuals. This result varied among orders (Characiformes $=7.4 \%$, Perciformes $=16.6 \%$ and Siluriformes $=$ $21.9 \%)$, and among species (0\% to $67.4 \%)$. Arrington et al. (2002) explains this phenomenon by particular life history 


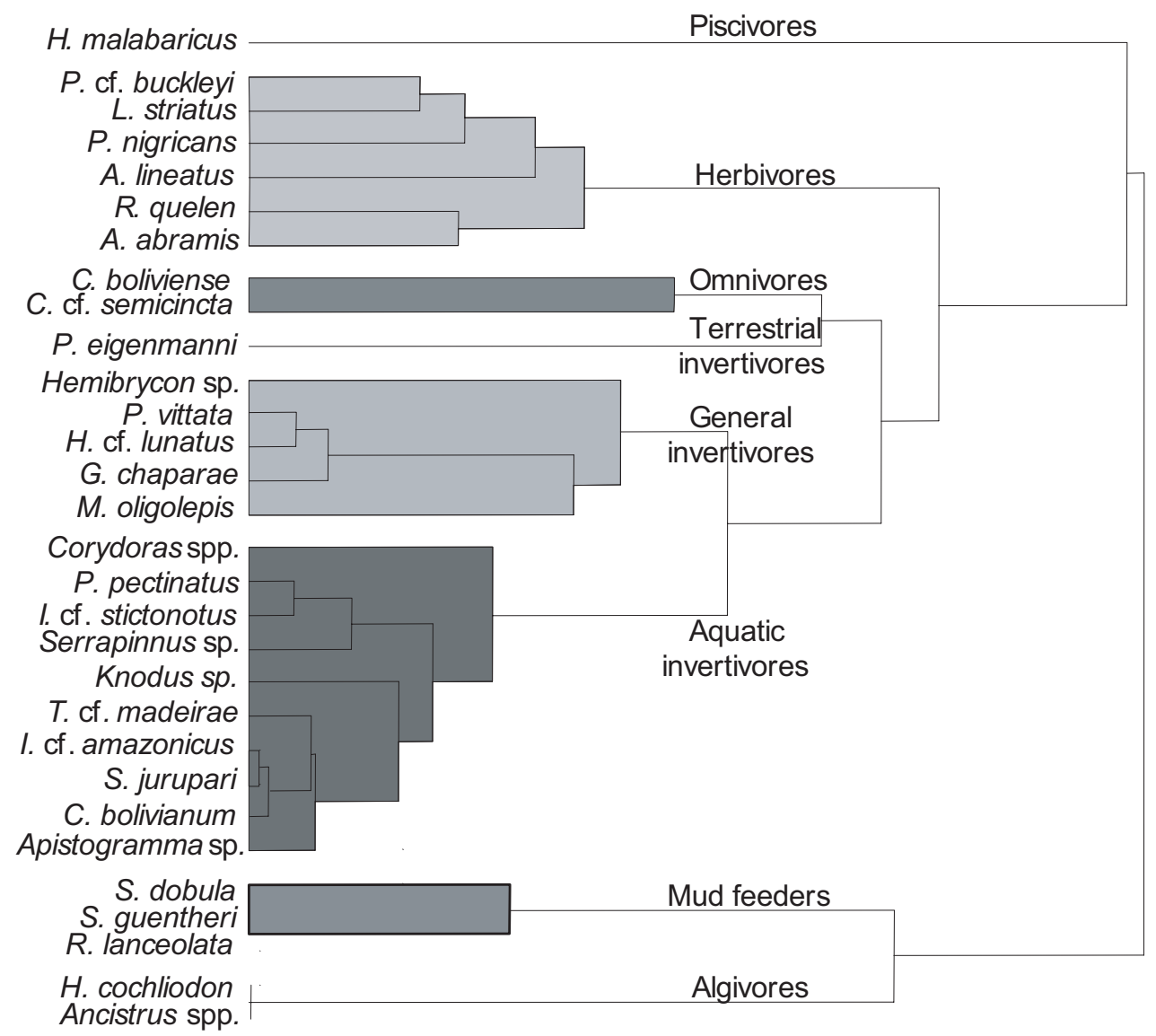

Fig. 2. Cluster analysis (UPGMA) showing trophic distances between the 30 species studied. Eight trophic guilds were distinguished: algivores, mud feeders, aquatic invertivores, general invertivores, terrestrial invertivores, omnivores, herbivores and piscivores.

traits related to diurnal or nocturnal activity of species associated with their trophic levels. Piscivorous species had the greater percentage of empty stomachs (e.g. $46.9 \%$ for Hoplias malabaricus) and algivorous species the smaller (e.g. $100 \%$ of full stomachs for Ancistrus spp. and Hypostomus gr. cochliodon).

Diet composition and abundance varied among the 30 species studied and our cluster analysis allowed us to identify eight broad trophic guilds (Table 4 and Fig. 2). The Piscivore guild was represented by only one species, Hoplias malabaricus (3.3\% of the total assemblage). Diet breadth (Levin's index, $B$ ) showed particularly high generalist tendency for $H$. malabaricus $(B=0.32)$. The Herbivorous guild was represented by six species accounting for $20 \%$ of the assemblage: Prochilodus nigricans, Parodon cf. buckleyi, Leporinus striatus, Astyanax lineatus, Rhamdia quelen and Astyanax abramis. Diet breadth reflected slight generalist tendency for $R$. quelen $(B=0.12)$ and $A$. abramis $(B=0.12)$ and high specialization tendency for $P$. buckleyi $(B=0.01)$. The other three species ranged between intermediate levels of specialization ( $B$ from 0.03 to 0.09$)$. Omnivores were represented by two species (6.6\% of the assemblage): Crenicichla cf. semicincta and $\mathrm{Ci}$ chlasoma boliviense, with generalist tendency $(B=0.37$ and $B=0.57$, respectively).

Invertivorous species were the dominant diet group representing $53.3 \%$ of the assemblage with 16 species. Our results distinguished between three groups of terrestrial, generalist and aquatic invertivores. Potamorrhaphis eigenmanni was found to be a terrestrial invertebrate specialist $(B=0.02)$. Five species (Pyrrhulina vittata, Hemibrycon sp., Hemigrammus cf. lunatus, Gephyrocharax chapare and Moenkhausia oligolepis) fed on both terrestrial and aquatic invertebrates, $(B$ varying between 0.02 and 0.26 ) and the third group (Phenacogaster pectinatus, Imparfinis cf stictonotus, Corydoras spp., Serrapinnus sp., Knodus sp., Tyttocharax cf. madeirae, Ituglanis cf. amazonicus, Satanoperca jurupari, Characidium bolivianum and Apistogramma sp.), specialized on aquatic invertebrates, presented high degree of specialization ( $B$ varying between 0 and 0.04).

Three species (representing 10\% of the assemblage) belonged to the mud feeder guild: Steindachnerina dobula, Steindachnerina guentheri and Rineloricaria lanceolata. These species showed highly specialized diet, with $B$ values between 0.00 and 0.02 . Finally, two species ( $6.6 \%$ of the assemblage) were grouped as algivorous, Ancistrus spp.and Hypostomus gr. cochliodon, with rather generalist tendencies $(B=0.16$ for both species).

\subsection{Phylogenetic constraints}

Except for one variable (i.e. gut length; GUTL), Moran's I coefficients showed no significant phylogenetic effect and no 


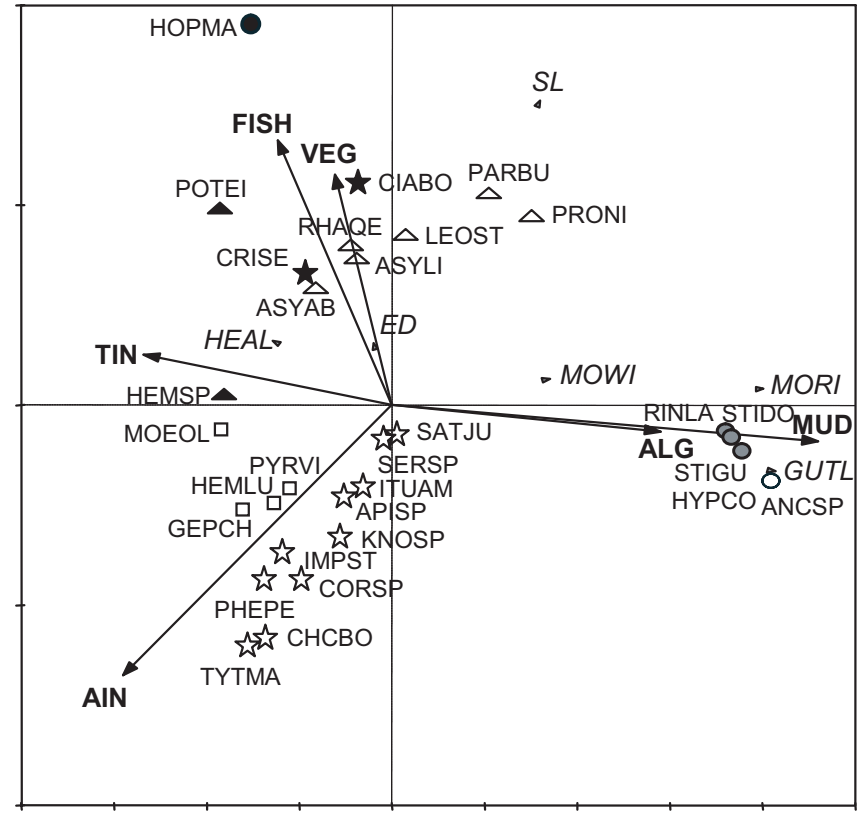

Fig. 3. Redundancy Analysis (RDA) linking species diets to morphology: algivores (open circle), mud feeders (gray closed circle), aquatic invertivores (open start), terrestrial invertivores (closed triangle), general invertivores (square), omnivores (closed start), herbivores (open triangle) and piscivores (closed circle).

regular decrease of autocorrelation with phylogenetic distance. Furthermore, concerning GUTL, the significant autocorrelation was positive instead of negative, as logically expected. Negative values suggest some kind of morphological convergence and in this case using methods to "remove" the phylogenetic effect would eliminate the desired data signal. As a result data in subsequent analyses were kept untransformed. The Moran's $I$ coefficient matrix including corresponding $\mathrm{p}$ values is available on request from the authors.

\subsection{Relationship between diet and morphology}

The first two axes of the RDA analysis relating diet to associated morphological variables accounted for $54.7 \%$ of the variation in species diets and for $95 \%$ of the variation explained by morphological variables (Fig. 3, Table 5). The global model was highly significant (Monte Carlo test ( $p=$ 0.001 ) (Table 5). Among the 6 morphological variables tested, only 3 were statistically significant $(p<0.001)$ : gut length (GUTL), mouth orientation (MORI) and standard length (SL). Axis 1 was positively related to GUTL and MORI while axis 2 was negatively related to SL (Table 5). Axis 1 (Fig. 3) clearly distinguished fish species feeding preferentially on algae and mud (e.g. Ancistrus spp., Steindachnerina guentheri, Steindachnerina dobula and Rineloricaria lanceolata) and displaying high relative gut length and an oblique or ventral mouth orientation. Axis 2 mainly separated a group of aquatic invertivores characterized by small size (Fig. 3 bottom-left), and a group of species showing a wide diversity of feeding habits (e.g. herbivorous, terrestrial invertivorous and piscivorous species; Fig. 3 top) and displaying larger body size.
Table 5. Results of redundancy analysis relating diet to associated morphological variables.

\begin{tabular}{lrr}
\hline Variable & Axis 1 Axis 2 \\
\hline Correlations of food items with ordination axes & & \\
TIN & $\mathbf{- 0 . 5 1 1}$ & 0.096 \\
AIN & $\mathbf{- 0 . 5 5 3}$ & $-\mathbf{0 . 5 1 1}$ \\
ALG & $\mathbf{0 . 5 5 2}$ & -0.049 \\
MUD & $\mathbf{0 . 8 7 7}$ & -0.069 \\
VEG & -0.118 & $\mathbf{0 . 4 3 6}$ \\
FISH & -0.235 & $\mathbf{0 . 5 0 0}$ \\
Summary statistics for ordination axes & & \\
Eigenvalues & 0.51 & 0.089 \\
Diet - morphological & & \\
correlations & 0.953 & 0.757 \\
Monte Carlo probability for significance of the sum of & \\
all eigenvalues (1000 permutations) $=0.0010$ & \\
\hline
\end{tabular}

\section{Discussion}

\subsection{Feeding habits and degree of specialization of species}

Thirty fish species were classified in eight trophic guilds: mud feeders, algivorous, aquatic invertivorous, general invertivorous, terrestrial invertivorous, omnivorous, herbivorous and piscivorous. Invertivorous species composed the dominant guild of this assemblage. This result is in agreement with the ones already obtained for other tropical (Angermeier and Karr 1983; Bojsen and Barriga 2002; Silva 1993; Uieda et al. 1997; Deus and Petrere-Junior 2003; Pouilly et al. 2006; Ibanez et al. 2007) and temperate forested streams (Schlosser 1982; Rahel and Hubert 1991; Oberdorff et al. 1993, 2002) suggesting a possible convergence in trophic structure between temperate and tropical fish assemblages (Ibañez et al. 2007). Trophic diversity of fish assemblages in such systems may be strongly related to food availability (Angermeier and Karr 1983), which in turn may be influenced by common environmental constraints. The organic energy base in forested headwater streams is essentially allochthonous and mostly comes from riparian vegetation through dead leaves, branches and wood processing by microbial and aquatic invertebrate communities (Wallace et al. 1997; Thompson and Townsend 2005; Tedesco et al. 2007). The energy flux reaching fishes thus strongly reflect the production of invertebrates via availability of terrestrial detritus and to a lesser extend aquatic primary production. It is thus coherent to expect a dominance of the invertivorous guild in these forested streams.

Our study strongly suggests a high degree of diet specialization for species at almost all trophic levels. In fact, if we arbitrarily adopt a quite conservative cut-off level at $\boldsymbol{B}<0.2$ (Levin's standardized index) for classifying a species as a specialist), $94 \%$ of the invertivorous guild (15 species), $100 \%$ of the herbivorous guild (6 species), $100 \%$ of mud feeders guild ( 2 species) and $100 \%$ of the algivorous guild ( 2 species) can be considered specialist feeders. Only omnivorous and piscivorous (only one species for the later) guilds 
were represented by more generalist species (this generalist tendency was obviously expected concerning omnivorous species). This result highlights the importance of feeding specialization in the segregation of trophic niches among species, as the probable explanation for the local maintenance of high species diversity in these highly diverse neotropical assemblages is via fine-scale niche partitioning by resource specialists (Tedesco et al. 2007).

\subsection{Phylogeny}

Although phylogenetic informations for neotropical freshwater fishes were scarce, we tried to account for evolutionary history of taxa by applying true phylogenetic distances available for $70 \%$ of the species analyzed (21 over a total of 30 species). No significant effect of phylogeny on species diets and morphology was found for this large subset of species. It is thus, very unlikely, that our final results are altered by spurious phylogenetical effects.

\subsection{Relationships between diet and morphology}

According to the RDA, fishes belonging to the algivorous and detritivorous guilds displayed large relative gut lengths. This appears to be a highly robust ecomorphological pattern since our finding is in agreement with results found in several other studies dealing with fish assemblages worldwide (Kotrschal 1989; Paugy 1994; Kramer and Bryant 1995; Hugueny and Pouilly 1999; Winemiller and Adite 1997; Delariva and Agostinho 2001; Pouilly et al. 2003; Ward-Campbell et al. 2005). Bowen (1983) working on neotropical fish communities ranked relative intestine development in relation to diet as: carnivorous $<$ omnivorous $<$ herbivorous $<$ detritivorous, which is generally interpreted as reflecting the resistance of different foods to digestion (Herder and Freyhof 2006). Our results are globally in accordance with this proposed scheme, as our ranking of relative intestine development in relation to trophic guilds, based on the RDA, was: invertivorous $<$ piscivorous $<$ herbivorous $<$ algivorous $<$ detritivorous (see Fig. 3). Benthic fishes from the algivorous and mud feeder guilds were also characterized by relatively narrow heads and a ventral (Loricaridae) or oblique (Curimatidae) mouth orientation and this result is again well supported by other studies (Gatz 1979; Watson and Balon 1984; Winemiller et al. 1995; Hugueny and Pouilly 1999; Pouilly et al. 2006). Fishes from the herbivorous and piscivorous guilds generally displayed a large standard length while species from the aquatic invertivorous guild were mostly characterized by their small sizes. These results accord, at least concerning invertivorous and piscivorous guilds, with the general observation that body and prey size are often correlated (Gatz 1979). The association between herbivory and large body size of species is difficult to interpret but has been also reported for other vertebrates such as lizards (Cooper and Vitt 2002).

Then, our results show that, independently of any phylogenetic constraints, some of the trophic guilds can be grossly predicted from few relevant morphological attributes. Such consistent pattern suggests a link between diet and morphology.
In other words, species having similar diet tend to converge to some extent on some morphological attributes. Nevertheless, this link mostly concerns, in our case, three trophic guilds over the six previously defined (i.e. invertivore, algivore and detritivore guilds) and three morphological attributes over the six actually tested (i.e. standard length, relative gut length and mouth orientation). This suggests that even if morphology may limit patterns of resource-use, these limits are broad enough to allow most fishes changing their choice of prey resources in response to local biotic and abiotic conditions. For example, Pouilly et al. (2006) have analyzed the trophic structure of fish species in other neotropical streams of the Beni River basin of the Bolivian Amazon. We compared the trophic status and the diet breath (using Levin's standardized index) of four species common to both studies (i.e. Characidium bolivianum, Crenicichla semicincta, Hoplias malabaricus and Rhamdia quelen) (see Appendix 2). The trophic status was similar for two species (Characidium boliviense was classified as aquatic invertivore and Hoplias malabaricus was classified as piscivore, in both studies) but significantly differed for the two others (Crenicichla semicincta was classified as piscivore in the Beni River system and omnivore in our study, and Rhamdia quelen was classified as aquatic insectivore in the Beni River system and herbivore in our study). In return, based on Levin's standardized index values, the four species' diet breath was overall similar in both studies (Appendix 2). In the same way, Fritz (1974) working on phylogenetically related species with similar morphology (genus Astyanax), showed that within a similar habitat but on different rivers, similar diets were found.

\subsection{Conclusion}

In summary, we found that the position of a species along the trophic dimension is related partly to its morphology, independently of any phylogenetic effect. In other words, some morphological constraints acting on a species determine its potential trophic niche. Nevertheless, its realized niche seems to depend also partly on the environment (i.e. food availability and its variability between habitats). Among the morphological variables tested here, relative intestinal length and mouth orientation appear the most useful descriptors of diet, with the direction of relative morphological variations reflecting diet quality. These two morphological characters could be thus useful to predict resource use and trophic structure of fish assemblages of forested neotropical streams, where fish species feeding habits information is rather scarce.

Acknowledgements. We are indebted to numerous colleagues for their active participation to field work, our thank to: Jose Alcoreza, Jimena Camacho, Edgar Goitia, Kelvin Herbas, Paola Luna, Mabel Maldonado, Antonieta Mollo, Nabor Moya and Mirtha Rivero. A special thank to Dr. Alexandre Hassanin who generously gave us much assistance and guidance with phylogenetic analyses. The project was supported by the Institut de Recherche pour le Développement (IRD), the Limnological Research Unit (ULRA) of the University Mayor de San Simon of Cochabamba, Bolivia and the Limnological Unit of the University Mayor de San Andres of La Paz, Bolivia. Two anonymous reviewers gave thoughtful comments on the manuscript. 


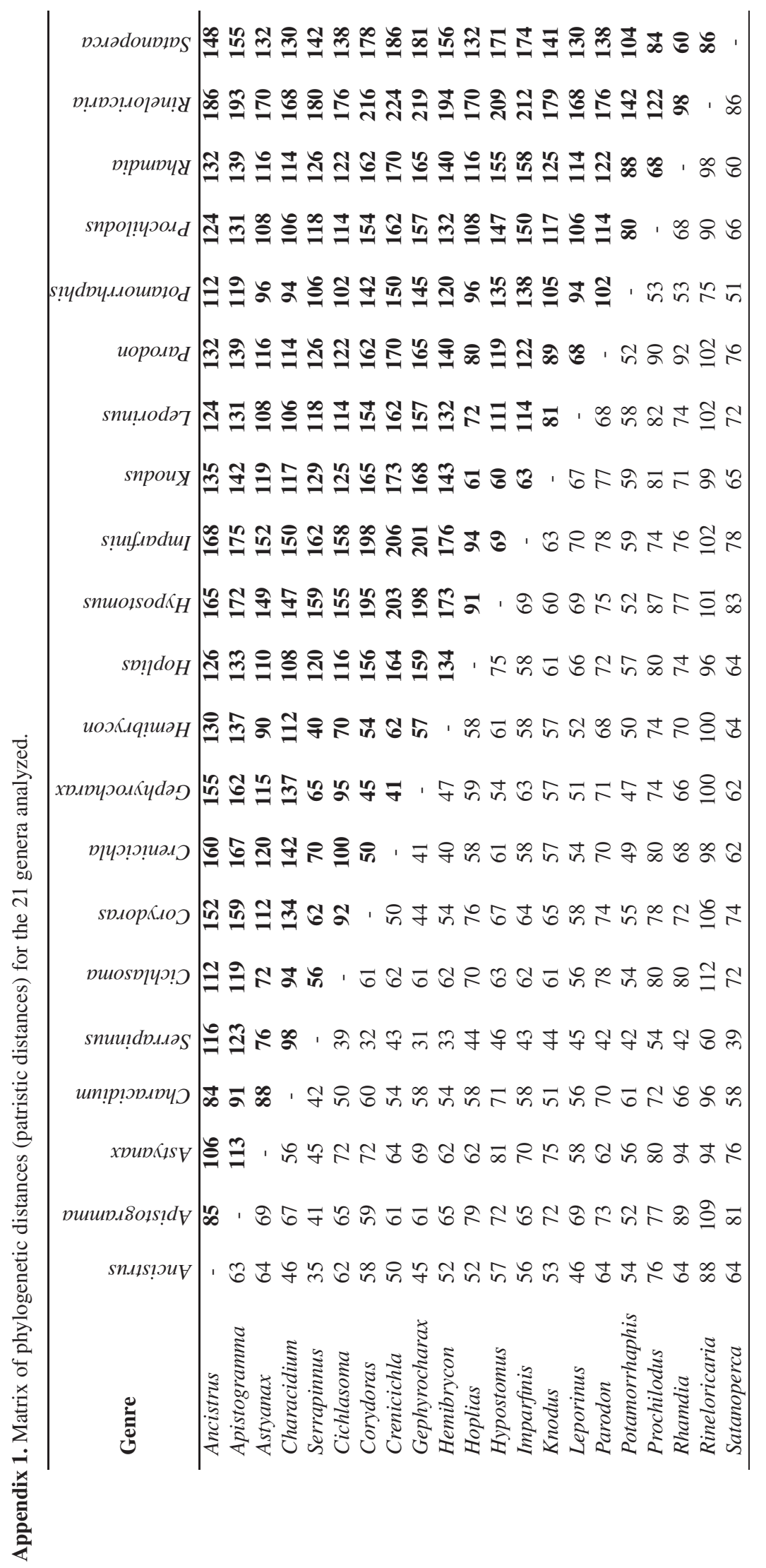


Appendix 2. Comparison of diet composition (\% of occurrence) and diet breadth (Lewin's index, B) of four species common to Pouilly et al. (2006) and the present study.

\begin{tabular}{lccccccccc}
\hline Species & Basin & Trophic & TIN & AIN & ALG & MUD & VEG & FISH & $\boldsymbol{B}$ \\
\hline Characidium bolivianum & Chapare & AIN & 0.03 & 1 & 0 & 0 & 0 & 0 & $\mathbf{0 . 0 0}$ \\
& Beni & AIN & 0.07 & 1 & 0.13 & 0.02 & 0.02 & 0 & $\mathbf{0 . 0 8}$ \\
Hoplias malabaricus & Chapare & OMN & 0.31 & 0.38 & 0 & 0.06 & 0.50 & 0.19 & $\mathbf{0 . 3 7}$ \\
& Beni & FISH & 0.03 & 0.67 & 0.05 & 0.03 & 0.05 & 0.38 & $\mathbf{0 . 2 0}$ \\
Crenicichla semicincta & Chapare & FISH & 0 & 0.47 & 0 & 0.06 & 0.65 & 0.47 & $\mathbf{0 . 3 2}$ \\
& Beni & FISH & 0 & 0.29 & 0 & 0.43 & 0.14 & 0.43 & $\mathbf{0 . 3 6}$ \\
Rhamdia quelen & Chapare & HER & 0.28 & 0.36 & 0.12 & 0.16 & 0.88 & 0.12 & $\mathbf{0 . 1 2}$ \\
& Beni & AIN & 0.25 & 0.96 & 0 & 0 & 0.11 & 0.14 & $\mathbf{0 . 1 6}$ \\
\hline
\end{tabular}

\section{References}

Angermeier P.L., Karr J.R., 1983, Fish communities along environmental gradients in a system of tropical streams. Environ. Biol. Fish. 9, 117-135.

Arrington D.A., Winemiller K.O., Loftus W.F., Akin S., 2002, How often do fishes Run on Empty. Ecology 83, 2145-2151.

Bellwood D.R., Wainwright P., Fulton C.J., Hoey A.S., 2006, Functional versatility supports coral reef biodiversity. Proc. R. Soc. B. 273, 101-107.

Bojsen B.H., Barriga R., 2002, Effects of deforestation on fish community structure in Ecuadorian Amazon streams. Freshw. Biol. 47, 2246-2260.

Bowen S.H., 1983, Detrivory in neotropical fish communities. Environ. Biol. Fishes 9, 137-144.

Chan M.D., 2001, Fish ecomorphology: Predicting habitat preferences of stream fishes from their body shape. PhD Thesis. Faculty of the Virginia Polytechnic Institute and State University, USA.

Cooper W.E., Vitt L.J., 2002, Distribution, extent, and evolution of plant consumption by lizards. J. Zool. 257, 487-517.

Delariva R., Agostinho A., 2001, Reationship between morphology and diets of six neotropical loricariids. J. Fish Biol. 58, 832-84.

Deus C.P., Petrere - Junior M., 2003, Seasonal diet shifts of seven fish species in an Atlantic Rainforest Stream in Southeastern Brazil. Braz. J. Biol. 64, 579-588.

Diogo R., 2003, Catfish morphology, phylogeny and macroevolution: A case study in theoretical phylogeny and macroevolution. Doctoral Thesis. Université de Liège.

Douglas M.E., Matthews W.J., 1992, Does morphology predict ecology? Hypothesis testing within a freshwater stream fish assemblage. Oikos 65, 213-224.

Elliott J.P., Bellwood D.R., 2003, Alimentary tract morphology and diet in three coral reef fish families. J. Fish Biol. 63, 1598-1609.

Felsenstein J., 1985, Phylogenies and the comparative method. Am. Nat. 125, 1-15.

Fritz E.S., 1974, Total diet comparison in fishes by Spearman rank correlation coefficients. Copeia 1, 210-214.

Frost W.E., Went A.E., 1940, River liffey survey III. The growth and food of young Salmon. Proc. R. Ir. Acad. 46, 53-80.

Gatz A.J., 1979, Ecological morphology of freshwater stream fishes. Tulane studies in zoology and botany 21, 91-124.

Gibson R.N., Ezzi A., 1987, Feeding relationships of a Demersal fish assemblage on the West Coast of Scotland. J. Fish Biol. 31, 5569.

Grossman G.D., 1986, Food resource partitioning in a rocky intertidal fish assemblage. J. Zool. 1, 317-355.

Harvey P.H., Pagel M.D., 1991, The comparative method in evolutionary biology. Oxford: Oxford University Press.
Hegrenes S., 2001, Diet-induced phenotypic plasticity of feeding morphology in the orangespotted sunfish, Lepomis humilis. Ecol. Freshw. Fish. 10, 35-42.

Herder F., Freyhof J., 2006, Resource partitioning in a tropical stream fish assemblage. J. Fish Biol. 69, 571-589.

Hugueny B., Pouilly, M., 1999, Morphological correlates of diet in an assemblage of West African freshwater fishes. J. Fish Biol. 54, 1310-1325.

Hurlbert S.H., 1978, The measurement of niche overlapand some relatives. Ecology 59, 67-77.

Hyslop E.J., 1980, Stomach contents analysis-a review of methods and their application. J. Fish Biol. 17, 411-429.

Ibañez C., Oberdorff T., Teugels G., Mamononekene V., Lavoué S., Fermon Y., Paugy D., Toham A.K., 2007, Fish assemblages structure and function along environmental gradients in rivers of Gabon Africa). Ecol. Freshw. Fish.

(doi: 10.1111/j.1600-0633.2006.00222.x).

Kramer D., Bryant M., 1995, Intestine length in the fishes of a tropical stream: 2. relationship to diet-the long and- short of a convoluted issue. Environ. Biol. Fishes 42, 129-141.

Kotrschal K., 1989, Trophic ecomorphology in eastern Pacific blennioid fishes: character transformation of oral jaws and associated change of their biological roles. Environ. Biol. Fishes 24, 199-218.

Legendre P., Legendre L., 1998, Numerical Ecology. Amsterdam: Elsevier.

Legendre P., Borcard D., Peres-Neto, P., 2005, Analyzing beta diversity: partitioning the spatial variation of community composition data. Ecol. Monogr. 75, 435-450.

Makarenkov V., Legendre P., 2002, Nonlinear redundancy analysis and canonical correspondence analysis based on polynomial regression. Ecology 83, 1146-1161.

Malabarba L.R., Reis R.E., Vari R.P., Lucena Z.M., Lucena C.A.S. (Eds), 1998, Phylogeny and classification of Neotropical fishes. Edipucrs, Porto Alegre.

Michel P., Oberdorff T., 1994, Feeding habits of fourteen European freshwater fish species. Cybium 19, 5-46.

Moran P.A.P., 1948, The interpretation of statistical maps. J. R. Stat. Soc. B. 10, 243-251.

Motta P.J., 1988, Functional morphology of the feeding apparatus of ten species of Pacific butterflyfishes Perciformes, (Chaetodontidae): An ecomorphological approach. Environ. Biol. Fishes 22, 39-67.

Navarro G., Maldonado M., 2002, Geografía Ecológica de Bolivia: Vegetación y Ambientes Acuáticos. Editorial: Centro de Ecología Simón I. Patiño-Departamento de difusión. Cochabamba, Bolivia.

Oberdorff T., Guilbert E., Luchetta J.C., 1993, Patterns of fish species richness in the Seine River basin, France. Hydrobiologia 259, 157-167. 
Oberdorff T., Pont D., Hugueny B., Porcher J.P., 2002, Development and validation of a fish-based index for the assessment of rivers "health" in France. Freshw. Biol. 47, 1720-1735.

Paugy D., 1994, Ecologie des poissons tropicaux d'un cours d'eau temporaire (Baoulé, haut bassin du Sénégal au Mali): Adaptation au milieu et plasticité du régime alimentaire. Rev. Hydrobiol. Trop. 27, 157-172.

Piet G., 1998, Ecomorphology of a size-structured tropical freshwater fish community. Environ. Biol. Fishes 51, 67-86.

Pouilly M., Lino F., Bretenoux G., Rosales C., 2003, Dietarymorphological relationships in a fish assemblage of the Bolivian Amazonian floodplain. J. Fish Biol. 62, 1137-1158.

Pouilly M., Barrera S., Rosales C., 2006, Changes of taxonomic and trophic structure of fish assemblages along an environmental gradient in the Upper Beni watershed Bolivia). J. Fish Biol. 68, 137156.

Power M.E., 1983, Grazing responses of tropical freshwater fishes to different scales of variation in their food. Environ. Biol. Fishes 9, 103-115.

Power M.E., 1984, The importance of sediment in the grazing ecology and size class interactions of an armored catfish, Ancistrus spinosus. Environ. Biol. Fishes 10, 173-181.

Rahel F.J., Hubert W.A., 1991, Fish assemblages and habitat gradients in a Rocky Mountain-Great Plains stream: biotic zonation and additive patterns of community change. Trans. Am. Fish. Soc. 120, 319-332.

Schlosser I.J., 1982, Fish community structure and function along two habitat gradients in a headwater stream. Ecol. Monogr. 52, 395414.

Schneider C.J., Smith T.B., Larison B., Moritz C., 1999, A test of alternative models of diversification in tropical rainforests: Ecological gradients vs. rainforest refugia. Proc. Natl. Acad. Sci. USA 96, 13869-13873.

Silva C.P.D., 1993, Alimentação e distribuição espacial de algumas espécies de peixes do igarapé do Candirú, Amazonas. Brasil. Acta Am. 23, 271-285.

Swofford D.L., 2002, PAUP. Phylogenetic Analysis Using Parsimony. Sunderland, M. A. Sineaur Associates.

Tedesco P.A., Ibañez C., Moya N., Bigorne R., Camacho J., Goitia E., Hugueny B., Maldonado M., Rivero M., Tomanová S., Zubieta J.P., Oberdorff T., 2007, Local scale species-energy relationships in tropical fish assemblages of some forested streams of the Bolivian Amazon. C.R. Biol. 330, 255-264.
Ter Braak C.J.F., Smilauer P., 1998, CANOCO reference manual and user's guide to CANOCO for Windows: software for canonical community ordination. Version 4. Microcomputer Power, Ithaca, New York, USA.

Thompson R.M., Townsend C.R., 2005, Energy availability, spatial heterogeneity and ecosystem size predict food-web structure in streams. Oikos 108, 137-148.

Tresierra A., Culquichicón Z.G., 1993, Biología pesquera. Ed. Universitaria. Trujillo, Perú.

Uieda V.S., Buzzato P., Kikushi R.M., 1997, Partilha de recursos alimentares em peixes em um riacho de serra do Sudeste do Brasil. Nat. Acad. Bras. Ci. 69, 243-252.

Wainwright P.C., Osemberg C.W., Mittelbach G.G., 1991, Trophic polymorphism in the pumpkinseed sunfish (Lepomis gibbosus Linnaeus): effects of environment on ontogeny. Funct. Ecol. 5, 40-55.

Wallace J.B., Eggert S.L., Meyer J.L., Webster J.R., 1997, Multiple trophic levels of a forest stream linked to terrestrial litter inputs. Science 277, 102-104.

Ward-Campbell B.M.S., Beamish F.W.H., Kongchaiya C., 2005, Morphological characteristics in relation to diet in five coexisting Thai fish species. J. Fish Biol. 67, 1266-1279.

Watson D.J., Balon E.K., 1984, Ecomorphological analysis of fish taxocenes in rainforest stream of northern Borneo. J. Fish Biol. 25, 371-384.

Wikramanayake E.D., 1990, Ecomorphology and biogeography of a tropical stream fish assemblage: Evolution of assemblage structure. Ecology 71, 1756-1764.

Wimberger P.H., 1992, Plasticity of fish body shape. The effects of diet, development, family and age in two species of Geophagus (Pisces, Cichlidae). Biol. J. Linn. Soc. 45, 197-218.

Winemiller K.O., 1991, Ecomorphological diversification in lowland freshwater fish assemblages from five biotic regions. Ecol. Monogr. 61, 343-365.

Winemiller K.O., Kelso-Winemiller L.C., Brenkert A.L., 1995, Ecomorphological diversification and convergence in fluvial cichlid fishes. Environ. Biol. Fishes 44, 235-261.

Winemiller K.O., Adite A., 1997, Convergent evolution of weakly electric fishes from floodplain habitats in Africa and South America. Environ. Biol. Fishes 49,175-186.

Xie S., Cut Y., Li Z., 2001, Dietary-morphological relationships of fishes in Liangzi Lake, China. J. Fish Biol. 58, 1714-1729. 